Assiut Scientific Nursing Journal

http://asnj.journals.ekb.eg

http://www.arabimpactfactor.com

\title{
The Effect of Acupressure on Chronic Low Back Pain among Operating Rooms Nurses
}

\author{
Wafaa Hassan Ali Awad ${ }^{1}$, Mervat Abdel Hamid Hawash ${ }^{2}$ \\ ${ }^{1 .}$ Lecturer of Medical Surgical Nursing Faculty of Nursing, Alexandria University, Egypt. \\ 2. Lecturer of Medical Surgical Nursing, Faculty of Nursing, Alexandria University, Egypt.
}

\begin{abstract}
:
Low back pain is the most common musculoskeletal work-related condition among nurses. Acupressure is the type of complementary medicine frequently used today. Acupressure is a technique that involves pressing with a finger to release endorphins, which causes muscle relaxation and pain relief. This study aimed at determining the effect of acupressure on chronic low back pain among nurses working in the operating rooms. Setting: This study was carried out in the operating rooms (OR) of three departments namely OR (A) for colon surgery, OR (B) for oncology\& hepatobiliary surgery. Finally OR (C) for head and neck surgery in Alexandria Main University Hospital. Design: a quasi-experimental design was used. Tools: Interviews scheduled to collect socio-demographic and job characteristics, Million visual analog scale (MVAS), and questionnaire were used for the occupational role. Results: a statistically significant difference in pain severity between the study and control group after 6 weeks of acupressure massage $(\mathrm{p}<0.001 *)$ was observed. Concerning the post-test mean score of occupational role in the study group was $19.50 \pm 8.22$, while in the control group was $52.33 \pm 7.42$, with a highly statistically significant difference between the two groups at $\mathrm{p}(0.001 *)$. Conclusion: Acupressure is an effective complementary therapy for reducing occupational low back pain in nurses employed in operating rooms as well as improving their occupational function. Recommendation: educational nursing program about effect and technique of acupressure should be conducted.
\end{abstract}

\section{Keywords :Acupressure, Chronic Low Back Pain, Nurses, Operating Room}

\section{Introduction:}

Nursing is a highly stressful career that has been ranked as the second professions in terms of physical activity with high rates of musculoskeletal disorders and Low Back Pain (LBP). (Sharafkhani, et al. 2014). Nurses constitute the largest group of healthcare providers, accounting for around 70\% (Movahedi, et al. 2017). Bono et al., 2001 and Movahedi et al., 2017 declared that nurses working in the operating theatre are high risk for LBP because their work environment predisposes them to various physical problems and stress. Also, they spend more time than other health care providers in providing direct care for patients, they have to walk and stand up during their shift most of the times for more than 6 hours a day (Tosunoz \& Oztunc, 2017). Low Back Pain (LBP) is the most prevalent musculoskeletal work-related disorder among nurses' and leads to extreme cognitive, sensory, physical, emotional, and developmental barriers for nurses ranging from $40 \%$ to $97.9 \%$ in frequency. Abou El-Soud et al., 2014, Tinubu et al 2010. According to the World Health Organization (WHO) 2007 studies, more than $80 \%$ of people will experience LBP over their lifetime, while about 4-33 $\%$ of the population suffers from it. Also, it is believed that adults in the working-age are the most affected with LBP, which is ranked as the highest and the top 10 diseases and injuries that cause disability worldwide than any other disease based on the evidence of Global Burden of Disease 2010. LBP involves multifactorial risk factors that can be categorized into individual and occupational risk factors and is represented into three types classified into acute, sub-acute, or chronic (Kamper et al., 2015; Tosunozet al 2017).

In this context, LBP management techniques differ significantly. As a management technique, many doctors rely on non-steroidal anti-inflammatory medications, neurotropic drugs, or steroid injections, antibiotics, and surgery (Salzberg \& Manusov 2013). Additionally, offering a non-pharmacological treatment for those who do not respond to medication, have medication side effects, or are unable to take medication. One of the most appropriate non-pharmacological methods of pain management is acupressure. It is an additional procedure that goes back 5000 years. It is derived 
from traditional Chinese medicine and is now known every day in the world (HuJohnson, 2007).

Acupressure is non-invasive procedure and is based on acupuncture's theoretical model, where Chi energy flows through channels known as meridians. Acu-points are a control points for the Chi energy flow and are found along the meridians. It can be rebalanced or re-stimulated if the energy flow in meridians is delayed, blocked, or hyper-stimulated by applying pressure (acupressure) or inserting a needle (acupuncture) into one or more of these acupoints. It involves manually applying gentle but strong pressure to meridian points which activates endorphin release that contributes to muscle relaxation, and pain relief. As a result, physical therapists and other clinicians have begun to use it to treat LBP with the assumption that eliminating obstructions that impede energy transfer and relieving pain by enhancing circulation and nutrition is the best way to treat LBP (Yeh CH et al., 2012 \& Chen YW et al., 2014).

As a non-invasive, cheap, relatively easy to practice, more available than medical techniques, acupressure has many distinct advantages.

This study aimed to evaluate the effect of acupressure sessions on relieving low back pain among operating room nurse. As nurses working in operating room, have serious incidents of occupational LBP. Acupressure is one of the complementary medicine frequently used today, the nurse should master the proper allocation methods of the selected acupoints and use finger pressured directly on the point for proper acupressure application (Bono JP et.al, 2001)

\section{Purpose of this study:}

Evaluate the effect of acupressure on chronic low back pain among operating room nurses.

\section{Research hypothesis:}

1- H1: Nurses who receive acupressure exhibit less back pain than those who don't receive it

2- H2: Nurses who receive acupressure exhibit a greater improvement in their occupational role than those who don't receive it

\section{Methodology}

Research Design: a quasi-experimental research design was used to conduct this study.

\section{Setting:}

This study was conducted in the operating rooms (OR) in three departments namely OR (A) for colon surgery, OR (B) for oncology \& hepatobiliary surgery. Finally OR $(C)$ for head and neck surgery at Alexandria Main University Hospital

\section{Subjects:}

- All available nurses working in the operating room either (scrub, circulating nurse , and anesthesia nurse) were involved in the study $(\mathrm{N}=$ 50 nurses)

- The study subjects were randomly divided into two equal groups control and experimental/acupressure).

- Acupressure Group: Nurses with chronic low back pain who received the acupressure technique

- Control Group: Nurses with chronic low back pain not received an intervention

\section{Inclusion criteria}

1- Age between 20-60 years.

2- Nurses willing to participate.

3- Free from previous back surgery or injury.

4- Not pregnant.

Tools: Three tools were utilized.

Tool I: A socio-demographic interview schedule:

It was developed by the researcher and includes 8 items about nurses' age, sex, educational level, marital status, number of children, years of experience, previous training about body mechanics and body mass index.

Tool II: Million visual analog scale (MVAS), This tool was developed by Million $R$ et al (1982). It is one of the most commonly used self-administered LBP questionnaires; it includes questions about (pain intensity, presence of stiffness and twisting), the effect of LBP on daily activities (sitting, standing, walking, and work) and social life. MVAS uses the Visual Analog Scale (VAS) which contains a standardized linear range from 0-10. The nurse was asked to place a mark indicating where the current back pain lies on the line. As 0 is "no pain", 1-3 represents "mild pain", 4-7 represents "moderate pain", 8-9 represents "severe pain", and 10 is the "worst possible pain".

Tool III: The occupational role questionnaire. This tool was developed by Kopec J (1998).This tool includes 8 questions about the effect of low back pain on nurses job as (work more slowly, nurses are less able to concentrate on work, take more frequent or longer breaks, nurses are more likely to lose a job, nurses have less satisfaction with job and need more help from co-workers) using a four point Likert scale (ranging from Not at all $=0$, A Little $=1$, 
Somewhat $=2$, A lot $=3$.

The scoring system of this tool ranged from $(8-40)$

where

Complete interference ranged from $(28-40)$

Partial interference range from (14 - 27)

Noninterference ranged from $(>13)$

\section{Method}

The study was implemented as follows:

1- An official permission was obtained from the Faculty of Nursing Alexandria University and the director of the three operating rooms and surgical director of the Main University Hospital. The permission was taken to facilitate the research implementation after explaining the aim of the study.

2- Written informed consent was taken from each nurse after explaining the nature and benefits of this research. The researcher emphasized that participation in the study was entirely voluntary and each nurse had the right to withdraw from the study at any time without giving any reason. As well, anonymity and confidentiality were ensured through coding the data.

3- Tools were tested for their content validity by five experts in the field of Medical-Surgical nursing at Alexandria University. Comments and suggestions were considered and the tools were modified accordingly. The validity for tool II was $97.5 \%$. Tool III was 0.92 , which means that tools were reliable.

4- The researcher received special training in the field of acupressure at the Faculty of Physical Education, Alexandria University.

5- A pilot study was carried out on $10 \%$ of nurses $(n=5)$ before implementing the actual study to ascertain the clarity and applicability of all study tools and to estimate the time required to complete the study questionnaires. In the light of the findings of the pilot study, modifications were done accordingly.

6- Data collection: After securing the administrative approval, the data was collected over 6 months from December 2019 to July 2020. The data was collected from the control group first, and then the study groups to prevent data contamination.

- Data was collected on the morning and the evening shift daily.

Around 7 nurses in morning shift and 4 nurses in evening shift, 2 sessions per week (total 12 sessions in 6 weeks).
- The researchers started by introducing themselves and explained the study aim.

- The researchers ensured that the place where the sessions were conducted is calm, with adequate lighting, well arranged, and comfortable.

- Tools filling took about 30-45 minutes.

7- Assessment of nurses socio-demographic and work characteristic, Million Visual Analog scale and effect of low back pain on the occupational role before acupressure technique

8- For Acupressure group: Acupressure included stimulating key points along the meridians of the body, called acupoints, to promote energy flow and correct imbalances of Chi energy. Using measuring tape, the researcher measured the cun measurement based on each nurse finger to assess the acupoint position. The nurses were asked to take a deep breath prior to intervention for the acupressure group and then applied the pressure on points bladder 60 (BL60) (behind the ankle joint, in the depression between bumps of the lateral malleolus and the Achilles tendon), 32 of the bladder (BL32) (on the second sacral foramen) and 30 of the gallbladder (GB30) (back of the leg between the greater trochanter and the sacral), in certain meridian diagrams, the acupressure point B54 or Bladder 54 is also numbered B40. This point is also called the Middle Crook and it is located in the middle of the crease in the back of the knee and GV4 or Governing Vessel 4 is another important acupressure point for lower back pain that can be found between the vertebrae at the waistline at the same level as acupressure point Bladder 23. 


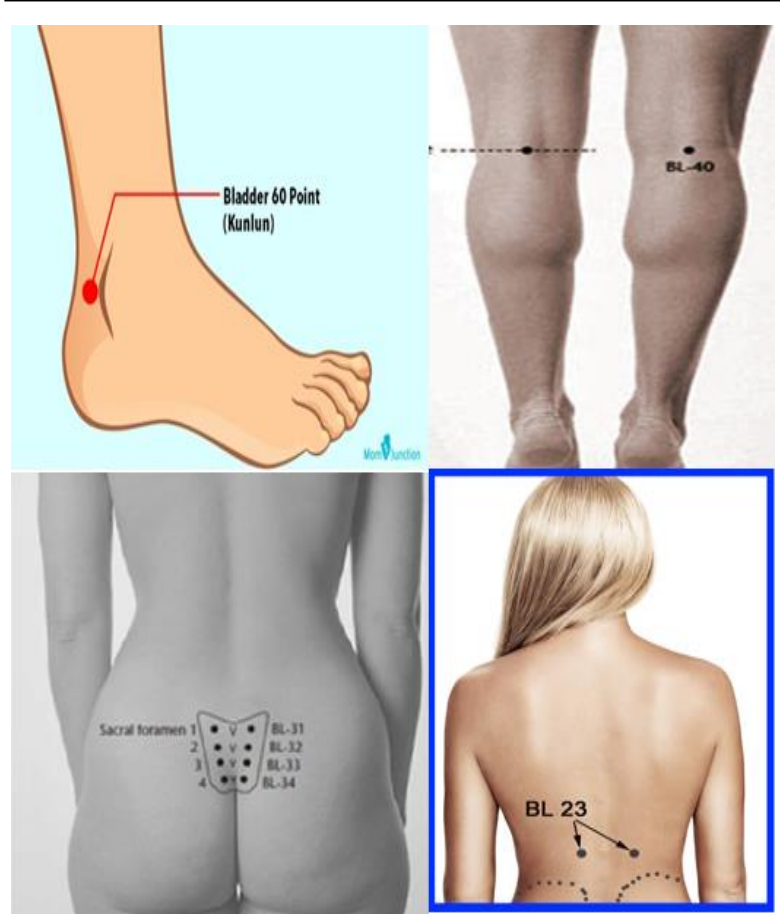

9- Pressure was applied to these main points for 1 minute (with a sequence of 4 seconds of pressure and 2 seconds of rest). Generally, pressure was applied on each point for 10 minutes on both sides, two times per week, during 6 weeks, totalizing 12 sessions, applied by a nurse for 30 minutes (Chen et al. 2015).The accuracy of the point was confirmed when the patient felt warmth, heaviness, swelling, or numbness at that point (Moemenabadi 2010; Chen et al. 2015; Dabiri\& Kaya,N (2019).

10-For the control group the nurses did not receive any intervention and were assessed for low back pain by tool I1 and tool III.

11-Follow up: The researcher assessed the low back pain and occupational role in both groups after 6 weeks. To keep contact with the study subjects, the researchers registered the phone numbers of each study subject.

\section{Ethical considerations}

1- Written consent was obtained from nurses after the aim of the study was explained.

2- Confidentiality and privacy of the collected data were secured.

3- Nurses right to withdraw at any time from the research was accepted and respected.

\section{Statistical Analysis}

Data were computerized and analyzed using IBM SPSS software package version 20.0. (Armonk, NY: IBM Corp) Qualitative data were described using number and percent. Quantitative data were described using range (minimum and maximum), mean, and standard deviation. Significance of the obtained results was accepted at the 5\% level.

\section{The tests used were}

1. Chi-square test: For categorical variables, to compare between different groups

2. Fisher's Exact or Monte Carlo correction: Correction for chi-square when more than $20 \%$ of the cells have expected count less than 5

3. Student t-test: For normally distributed quantitative variables, to compare between two studied groups

4. ANOVA with repeated measures: For normally distributed quantitative variables, to compare between more than two periods or stages, and Post Hoc test (Bonferroni adjusted) for pairwise comparisons

5. Friedman test: For abnormally distributed quantitative variables, to compare between more than two periods or stages and Post Hoc Test (Dunn's) for pairwise comparisons. 


\section{Results:}

Table (1): Comparison between the two studied groups according to socio demographic characteristics

\begin{tabular}{|c|c|c|c|c|c|}
\hline \multirow[t]{2}{*}{ Socio demographic characteristics } & \multicolumn{2}{|c|}{$\begin{array}{l}\text { Control group } \\
(\mathbf{n = 2 5})\end{array}$} & \multicolumn{2}{|c|}{$\begin{array}{l}\text { Study group } \\
\quad(n=25)\end{array}$} & \multirow{2}{*}{$\begin{array}{c}\text { Test of sig. } \\
\text { (p-value) }\end{array}$} \\
\hline & No. & $\%$ & No. & $\%$ & \\
\hline $\begin{array}{l}\text { Sex } \\
\text { Male } \\
\text { Female } \\
\end{array}$ & $\begin{array}{c}5 \\
20 \\
\end{array}$ & $\begin{array}{l}20.0 \\
80.0\end{array}$ & $\begin{array}{c}2 \\
23\end{array}$ & $\begin{array}{c}8.0 \\
92.0\end{array}$ & $\begin{aligned} \chi^{2} & =1.495 \\
(\mathrm{FE} & =0.417)\end{aligned}$ \\
\hline $\begin{array}{l}\text { Age (years) } \\
20-<30 \\
30-<40 \\
40-<50 \\
50-<60\end{array}$ & $\begin{array}{c}6 \\
15 \\
4 \\
0\end{array}$ & $\begin{array}{c}24.0 \\
60.0 \\
16.0 \\
0.0\end{array}$ & $\begin{array}{c}3 \\
19 \\
2 \\
1\end{array}$ & $\begin{array}{c}12.0 \\
76.0 \\
8.0 \\
4.0\end{array}$ & $\begin{array}{c}\chi^{2}=3.022 \\
\left({ }^{\mathrm{MC}} \mathrm{p}=0.359\right)\end{array}$ \\
\hline $\begin{array}{l}\text { Mean } \pm \text { SD } \\
\text { Min }- \text { Max }\end{array}$ & \multicolumn{2}{|c|}{$\begin{array}{c}33.68 \pm 7.26 \\
20.0-49.0\end{array}$} & \multicolumn{2}{|c|}{$\begin{array}{c}33.96 \pm 6.59 \\
20.0-51.0\end{array}$} & $\begin{array}{l}t=0.143 \\
(0.887)\end{array}$ \\
\hline $\begin{array}{l}\text { Education } \\
\text { Diploma } \\
\text { Technical } \\
\text { Bachelor } \\
\end{array}$ & $\begin{array}{c}17 \\
6 \\
2 \\
\end{array}$ & $\begin{array}{c}68.0 \\
24.0 \\
8.0\end{array}$ & $\begin{array}{c}20 \\
2 \\
3 \\
\end{array}$ & $\begin{array}{c}80.0 \\
8.0 \\
12.0 \\
\end{array}$ & $\begin{array}{c}\chi^{2}=2.401 \\
\left({ }^{\mathrm{MC}} \mathrm{p}=0.339\right)\end{array}$ \\
\hline $\begin{array}{l}\text { Marital } \\
\text { Single } \\
\text { Married } \\
\text { Divorced } \\
\text { Widow } \\
\end{array}$ & $\begin{array}{c}8 \\
14 \\
2 \\
1\end{array}$ & $\begin{array}{c}32.0 \\
56.0 \\
8.0 \\
4.0 \\
\end{array}$ & $\begin{array}{c}4 \\
19 \\
0 \\
2 \\
\end{array}$ & $\begin{array}{c}16.0 \\
76.0 \\
0.0 \\
8.0 \\
\end{array}$ & $\begin{array}{c}\chi^{2}=4.072 \\
\left({ }^{\mathrm{MC}} \mathrm{p}=0.253\right)\end{array}$ \\
\hline $\begin{array}{l}\text { Shift } \\
\text { Morning } \\
\text { Evening }\end{array}$ & $\begin{array}{c}16 \\
9\end{array}$ & $\begin{array}{l}64.0 \\
36.0\end{array}$ & $\begin{array}{c}20 \\
5\end{array}$ & $\begin{array}{l}80.0 \\
20.0\end{array}$ & $\begin{array}{c}\chi^{2}=1.587 \\
(0.208)\end{array}$ \\
\hline $\begin{array}{l}\text { Number of Child } \\
\text { No } \\
1-2 \\
3-4\end{array}$ & $\begin{array}{l}9 \\
8 \\
8\end{array}$ & $\begin{array}{l}36.0 \\
32.0 \\
32.0\end{array}$ & $\begin{array}{c}4 \\
7 \\
14\end{array}$ & $\begin{array}{l}16.0 \\
28.0 \\
56.0\end{array}$ & $\begin{array}{c}\chi^{2}=3.626 \\
(0.163)\end{array}$ \\
\hline $\begin{array}{l}\text { Experience (years) } \\
0-5 \\
6-10 \\
11-15 \\
16 \text { and more }\end{array}$ & $\begin{array}{c}6 \\
16 \\
3 \\
0 \\
\end{array}$ & $\begin{array}{c}24.0 \\
64.0 \\
12.0 \\
0.0\end{array}$ & $\begin{array}{c}2 \\
18 \\
3 \\
2 \\
\end{array}$ & $\begin{array}{c}8.0 \\
72.0 \\
12.0 \\
8.0 \\
\end{array}$ & $\begin{array}{c}\chi^{2}=3.725 \\
\left({ }^{\mathrm{MC}} \mathrm{p}=0.292\right)\end{array}$ \\
\hline $\begin{array}{l}\text { Safety training } \\
\text { Yes } \\
\text { No }\end{array}$ & $\begin{array}{c}2 \\
23\end{array}$ & $\begin{array}{c}8.0 \\
92.0\end{array}$ & $\begin{array}{c}4 \\
21 \\
\end{array}$ & $\begin{array}{l}16.0 \\
84.0\end{array}$ & $\begin{array}{c}\chi^{2}=0.758 \\
(\mathrm{FE} p=0.667)\end{array}$ \\
\hline $\begin{array}{l}\text { Body mass index }\left(\mathbf{k g} / \mathbf{m}^{2}\right) \\
\text { Under weight } \\
\text { Normal weight } \\
\text { Over weight } \\
\text { Obese } \\
\end{array}$ & $\begin{array}{c}3 \\
10 \\
10 \\
2 \\
\end{array}$ & $\begin{array}{c}12.0 \\
40.0 \\
40.0 \\
8.0 \\
\end{array}$ & $\begin{array}{c}2 \\
18 \\
3 \\
2 \\
\end{array}$ & $\begin{array}{c}8.0 \\
72.0 \\
12.0 \\
8.0 \\
\end{array}$ & $\begin{array}{c}\chi^{2}=6.325 \\
\left({ }^{\mathrm{MC}} \mathrm{p}=0.085\right)\end{array}$ \\
\hline
\end{tabular}

$\chi^{2}$ : Chi square test MC: Monte Carlo FE: Fisher Exact t: Student t-test

$\mathrm{p}$ : $\mathrm{p}$ value for comparing between the two studied groups

*: Statistically significant at $\mathrm{p} \leq 0.05$ 
Table (2): Comparison between the two studied groups according to visual analog pain assessment

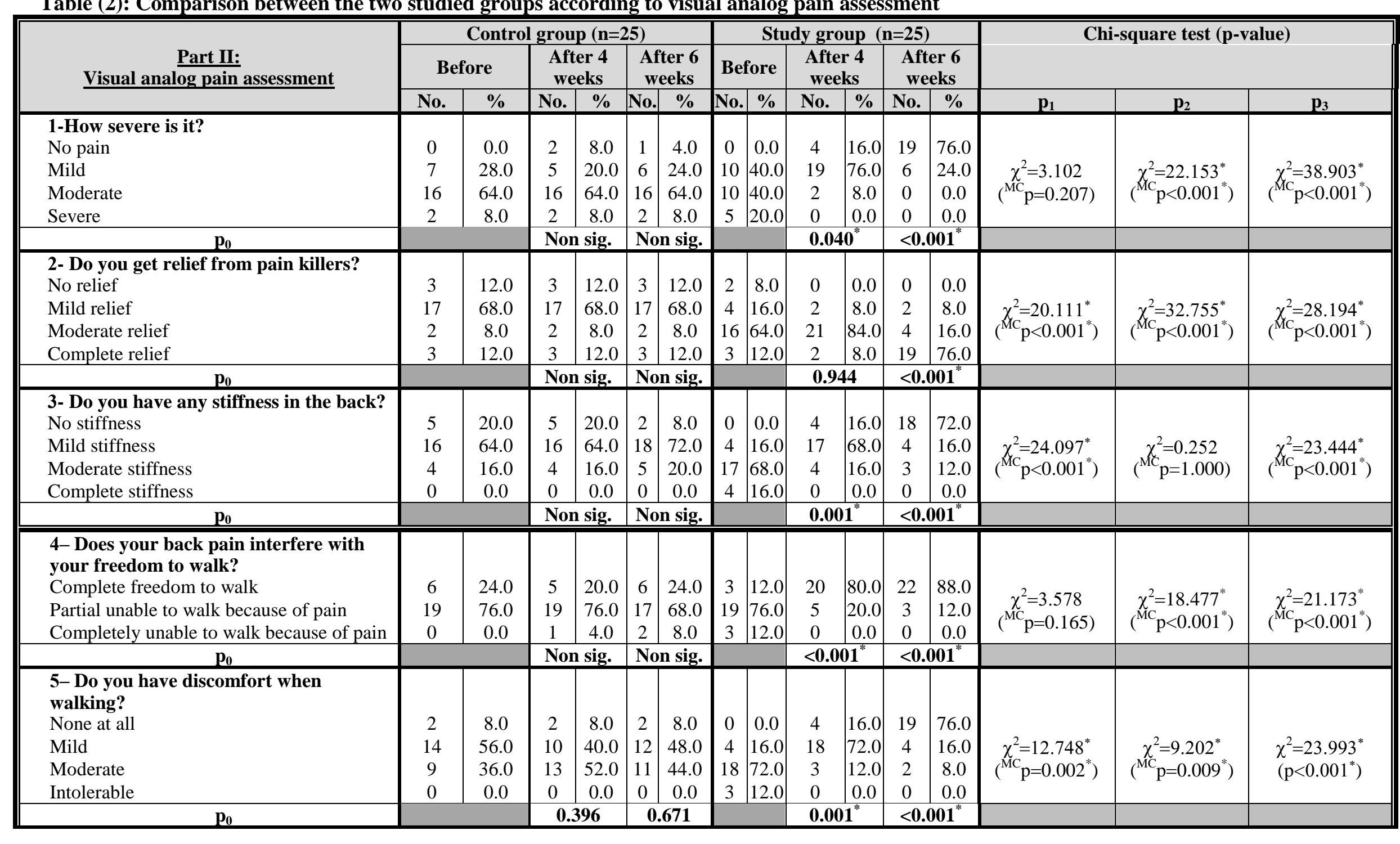


Abd-allah et al.,

\begin{tabular}{|c|c|c|c|c|c|c|c|c|c|c|c|c|c|c|c|}
\hline \multirow{3}{*}{$\begin{array}{c}\text { Part II: } \\
\text { Visual analog pain assessment }\end{array}$} & \multicolumn{6}{|c|}{ Control group $(n=25)$} & \multicolumn{6}{|c|}{ Study group $(n=25)$} & \multirow{2}{*}{\multicolumn{3}{|c|}{ Chi-square test (p-value) }} \\
\hline & \multicolumn{2}{|c|}{ Before } & \multicolumn{2}{|c|}{$\begin{array}{c}\text { After } 4 \\
\text { weeks }\end{array}$} & \multicolumn{2}{|c|}{$\begin{array}{c}\text { After } 6 \\
\text { weeks }\end{array}$} & \multicolumn{2}{|c|}{ Before } & \multicolumn{2}{|c|}{$\begin{array}{c}\text { After } 4 \\
\text { weeks }\end{array}$} & \multicolumn{2}{|c|}{$\begin{array}{c}\text { After } 6 \\
\text { weeks }\end{array}$} & & & \\
\hline & No. & $\%$ & No. & $\%$ & No. & $\%$ & No. & $\%$ & No. & $\%$ & No. & $\%$ & $p_{1}$ & $\mathbf{p}_{2}$ & $\mathbf{p}_{3}$ \\
\hline $\begin{array}{l}\text {-6 Does your pain interfere with your } \\
\text { ability to stand still? } \\
\text { Not able to stand still sometime } \\
\text { Stand still for a long time, that is an hour } \\
\text { Not able to stand still at all }\end{array}$ & $\begin{array}{c}25 \\
0 \\
0\end{array}$ & $\begin{array}{c}100.0 \\
0.0 \\
0.0 \\
\end{array}$ & $\begin{array}{c}21 \\
4 \\
0 \\
\end{array}$ & \begin{tabular}{|c|}
84.0 \\
16.0 \\
0.0 \\
\end{tabular} & \begin{tabular}{|c|}
22 \\
3 \\
0 \\
\end{tabular} & $\begin{array}{c}88.0 \\
12.0 \\
0.0 \\
\end{array}$ & \begin{tabular}{|c|}
3 \\
17 \\
5 \\
\end{tabular} & $\begin{array}{l}12.0 \\
68.0 \\
20.0\end{array}$ & $\begin{array}{c}19 \\
6 \\
0 \\
\end{array}$ & \begin{tabular}{|c|}
76.0 \\
24.0 \\
0.0 \\
\end{tabular} & $\begin{array}{c}21 \\
4 \\
0 \\
\end{array}$ & \begin{tabular}{|c|}
84.0 \\
16.0 \\
0.0 \\
\end{tabular} & $\begin{array}{c}\chi^{2}=43.986^{*} \\
\left({ }^{\mathrm{MC}} \mathrm{p}<0.001^{*}\right)\end{array}$ & $\begin{array}{l}\chi^{2}=0.500 \\
(\mathrm{p}=0.480)\end{array}$ & $\begin{aligned} \chi^{2} & =0.166 \\
\left({ }^{\mathrm{FE}} \mathrm{p}\right. & =1.000)\end{aligned}$ \\
\hline $\mathbf{p}_{0}$ & & & \multicolumn{2}{|c|}{ Non sig. } & \multicolumn{2}{|c|}{ Non sig. } & & & \multicolumn{2}{|c|}{$<0.001^{*}$} & \multicolumn{2}{|c|}{$<0.001^{*}$} & & & \\
\hline $\mathbf{p}_{0}$ & & & \multicolumn{2}{|c|}{\begin{tabular}{|l|} 
Non sig. \\
\end{tabular}} & \multicolumn{2}{|c|}{\begin{tabular}{|l|} 
Non sig. \\
\end{tabular}} & & & \multicolumn{2}{|c|}{$<0.001^{*}$} & \multicolumn{2}{|c|}{$<0.001^{*}$} & & & \\
\hline $\begin{array}{l}\text { 9- Does your back pain allow you to sit on } \\
\text { an upright hard chair? } \\
\text { Complete freedom to sit on a hard chair } \\
\text { So much pain that cannot sit on such a chair } \\
\text { at all }\end{array}$ & $\begin{array}{c}4 \\
21\end{array}$ & $\begin{array}{l}16.0 \\
84.0\end{array}$ & $\begin{array}{c}0 \\
25\end{array}$ & $\begin{array}{c}0.0 \\
100.0\end{array}$ & $\begin{array}{c}0 \\
25\end{array}$ & $\begin{array}{c}0.0 \\
100.0\end{array}$ & $\left|\begin{array}{c}5 \\
20\end{array}\right|$ & $\begin{array}{l}20.0 \\
80.0\end{array}$ & $\begin{array}{c}19 \\
6\end{array}$ & $\left|\begin{array}{l}76.0 \\
24.0\end{array}\right|$ & $\begin{array}{c}21 \\
4\end{array}$ & $\begin{array}{l}84.0 \\
16.0\end{array}$ & $\begin{aligned} & \chi^{2}=0.136 \\
&\left({ }^{\mathrm{E}} \mathrm{p}=1.000\right)\end{aligned}$ & $\begin{array}{l}\chi^{2}=30.645^{*} \\
\left(p<0.0011^{*}\right)\end{array}$ & $\begin{array}{l}\chi^{2}=36.207^{*} \\
\left(\mathrm{p}<0.001^{*}\right)\end{array}$ \\
\hline$\underline{\mathbf{p}_{0}}$ & & & \multicolumn{2}{|c|}{\begin{tabular}{|l} 
Non sig. \\
\end{tabular}} & \multicolumn{2}{|c|}{ Non sig. } & & & \multicolumn{2}{|c|}{$0.0166^{*}$} & \multicolumn{2}{|c|}{$<0.001^{*}$} & & & \\
\hline $\begin{array}{l}\text { 11- What is your overall handicap in your } \\
\text { complete lifestyle because of back pain? } \\
\text { Completely free to perform any task } \\
\text { Mild handicapped to perform any task } \\
\text { Moderate handicapped to perform any task } \\
\text { Totally handicapped }\end{array}$ & $\begin{array}{c}12 \\
9 \\
4 \\
0\end{array}$ & \begin{tabular}{|c|}
48.0 \\
36.0 \\
16.0 \\
0.0 \\
\end{tabular} & $\begin{array}{c}12 \\
10 \\
3 \\
0\end{array}$ & \begin{tabular}{|c|}
48.0 \\
40.0 \\
12.0 \\
0.0
\end{tabular} & $\begin{array}{c}13 \\
9 \\
3 \\
0\end{array}$ & \begin{tabular}{|c|}
52.0 \\
36.0 \\
12.0 \\
0.0
\end{tabular} & \begin{tabular}{|c|}
2 \\
5 \\
18 \\
0 \\
\end{tabular} & $\begin{array}{c}8.0 \\
20.0 \\
72.0 \\
0.0\end{array}$ & $\begin{array}{c}6 \\
19 \\
0 \\
0\end{array}$ & $\begin{array}{c}24.0 \\
76.0 \\
0.0 \\
0.0\end{array}$ & $\begin{array}{l}21 \\
4 \\
0 \\
0\end{array}$ & $\begin{array}{c}84.0 \\
16.0 \\
0.0 \\
0.0\end{array}$ & $\begin{array}{c}\chi^{2}=17.195^{*} \\
\left(<0.001^{*}\right)\end{array}$ & $\begin{array}{c}\chi^{2}=7.310^{*} \\
\left({ }^{\mathrm{MC}} \mathrm{p}=0.013^{*}\right)\end{array}$ & $\begin{array}{c}\chi^{2}=6.318^{*} \\
\left({ }^{\mathrm{MC}} \mathrm{p}=0.030^{*}\right)\end{array}$ \\
\hline $\mathbf{p}_{0}$ & & & Nor & sig. & Nol & $n$ sig. & & & 0.0 & & $<0$. & $001^{*}$ & & & \\
\hline
\end{tabular}


Abd-allah et al.,

\begin{tabular}{|c|c|c|c|c|c|c|c|c|c|c|c|c|c|c|c|}
\hline \multirow{3}{*}{$\begin{array}{c}\text { Part II: } \\
\text { Visual analog pain assessment } \\
\end{array}$} & \multicolumn{6}{|c|}{ Control group (n=25) } & \multicolumn{6}{|c|}{ Study group $(\mathbf{n}=25)$} & \multirow{2}{*}{\multicolumn{3}{|c|}{ Chi-square test (p-value) }} \\
\hline & \multicolumn{2}{|c|}{ Before } & \multicolumn{2}{|c|}{$\begin{array}{c}\text { After } 4 \\
\text { weeks }\end{array}$} & \multicolumn{2}{|c|}{$\begin{array}{c}\text { After } 6 \\
\text { weeks }\end{array}$} & \multicolumn{2}{|c|}{ Before } & \multicolumn{2}{|c|}{$\begin{array}{c}\text { After } 4 \\
\text { weeks }\end{array}$} & \multicolumn{2}{|c|}{$\begin{array}{l}\text { After } 6 \\
\text { weeks }\end{array}$} & & & \\
\hline & No. & $\%$ & No. & $\%$ & No. & $\%$ & No. & $\%$ & No. & $\%$ & No. & $\%$ & $\mathbf{p}_{1}$ & $\mathbf{p}_{2}$ & $p_{3}$ \\
\hline $\begin{array}{l}\text { 12- To what extent does your pain } \\
\text { interfere with your work? } \\
\text { No interference at all } \\
\text { Sometime interference } \\
\text { Totally incapable of work }\end{array}$ & $\begin{array}{c}10 \\
15 \\
0 \\
\end{array}$ & $\begin{array}{c}40.0 \\
60.0 \\
0.0 \\
\end{array}$ & $\begin{array}{c}10 \\
15 \\
0 \\
\end{array}$ & $\begin{array}{c}40.0 \\
60.0 \\
0.0 \\
\end{array}$ & $\begin{array}{c}10 \\
15 \\
0 \\
\end{array}$ & $\begin{array}{c}40.0 \\
60.0 \\
0.0 \\
\end{array}$ & \begin{tabular}{|c|}
0 \\
20 \\
5 \\
\end{tabular} & $\begin{array}{c}0.0 \\
80.0 \\
20.0 \\
\end{array}$ & $\begin{array}{c}16 \\
9 \\
0 \\
\end{array}$ & \begin{tabular}{|c}
64.0 \\
36.0 \\
0.0 \\
\end{tabular} & $\begin{array}{c}22 \\
3 \\
0 \\
\end{array}$ & $\begin{array}{c}88.0 \\
12.0 \\
0.0 \\
\end{array}$ & $\begin{array}{l}\chi^{2}=16.680^{*} \\
\left({ }^{\mathrm{MC}} \mathrm{p}<0.001^{*}\right)\end{array}$ & $\begin{aligned} & \chi^{2}=2.885 \\
&(0.089)\end{aligned}$ & $\begin{array}{c}\chi^{2}=12.500^{*} \\
\left(<0.001^{*}\right)\end{array}$ \\
\hline & & & \multicolumn{2}{|c|}{ Non sig. } & \multicolumn{2}{|c|}{\begin{tabular}{|l|} 
Non sig. \\
\end{tabular}} & & & \multicolumn{2}{|c|}{$<0.001^{*}$} & \multicolumn{2}{|c|}{$<0.001^{*}$} & & & \\
\hline $\begin{array}{l}\text { 13- To what extent does your work have } \\
\text { to be modified so that you are able to do } \\
\text { your job? } \\
\text { No adjustment to work } \\
\text { So much adjustment that you have had to } \\
\text { change your job }\end{array}$ & $\begin{array}{c}25 \\
0\end{array}$ & $\begin{array}{c}100.0 \\
0.0\end{array}$ & $\begin{array}{c}24 \\
1\end{array}$ & $\begin{array}{l}96.0 \\
4.0\end{array}$ & $\begin{array}{c}21 \\
4 \\
\end{array}$ & $\begin{array}{l}84.0 \\
16.0\end{array}$ & $\begin{array}{c}9 \\
16\end{array}$ & $\begin{array}{l}36.0 \\
64.0\end{array}$ & $\begin{array}{c}21 \\
4 \\
\end{array}$ & $\begin{array}{l}84.0 \\
16.0\end{array}$ & $\begin{array}{c}22 \\
3 \\
\end{array}$ & $\begin{array}{l}88.0 \\
12.0\end{array}$ & $\begin{array}{c}\chi^{2}=23.529^{*} \\
\left(<0.001^{*}\right)\end{array}$ & $\begin{array}{c}\mathrm{XE}_{\mathrm{X}} \chi^{2}=2.0 \\
\mathrm{p}=0.349)\end{array}$ & $\begin{array}{c}\chi^{2}=0.166 \\
\left({ }^{\mathrm{F}} \mathrm{p}=1.000\right)\end{array}$ \\
\hline $\mathbf{p}_{0}$ & & & \multicolumn{2}{|c|}{ Non sig. } & \multicolumn{2}{|c|}{\begin{tabular}{|l|} 
Non sig. \\
\end{tabular}} & & & \multicolumn{2}{|c|}{$0.011^{*}$} & \multicolumn{2}{|c|}{$0.006 *$} & & & \\
\hline
\end{tabular}

\section{$\chi^{2}$ : Chi square test MC: Monte Carlo FE: Fisher Exact}

$\mathrm{p}_{0}$ : $\mathrm{p}$-value for value for comparing between before and each other period

$\mathrm{p}_{1}$ : $\mathrm{p}$-value comparing between control group and study group before

$\mathrm{p}_{2}$ : $\mathrm{p}$-value comparing between control group and study group after 4 weeks

$\mathrm{p}_{3}$ : $\mathrm{p}$-value comparing between control group and study group after 6 weeks

*: Statistically significant at $\mathrm{p} \leq 0.05$ 
Table (3): Comparison between the two studied groups according to the occupational role questionnaire

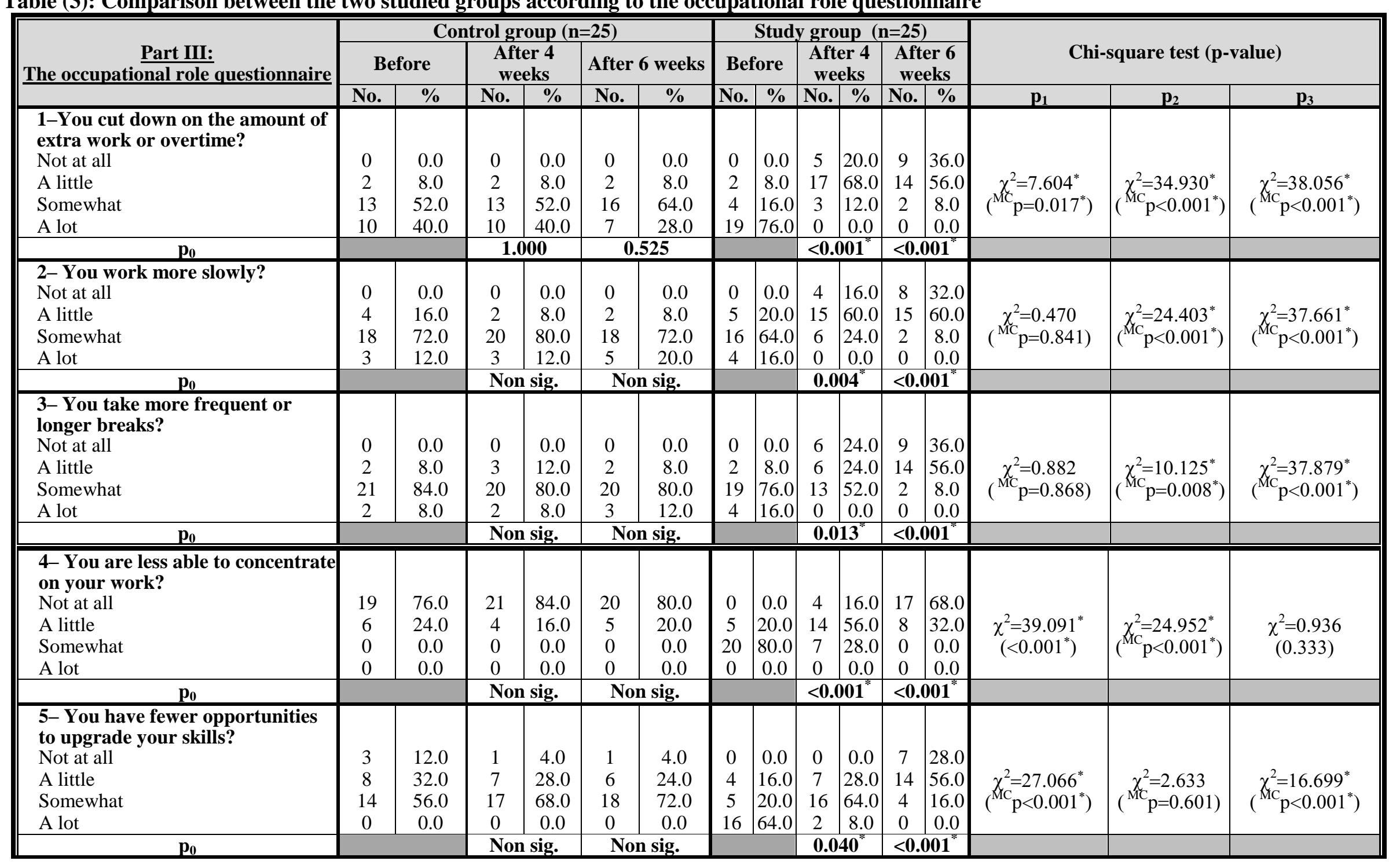


Abd-allah et al.,

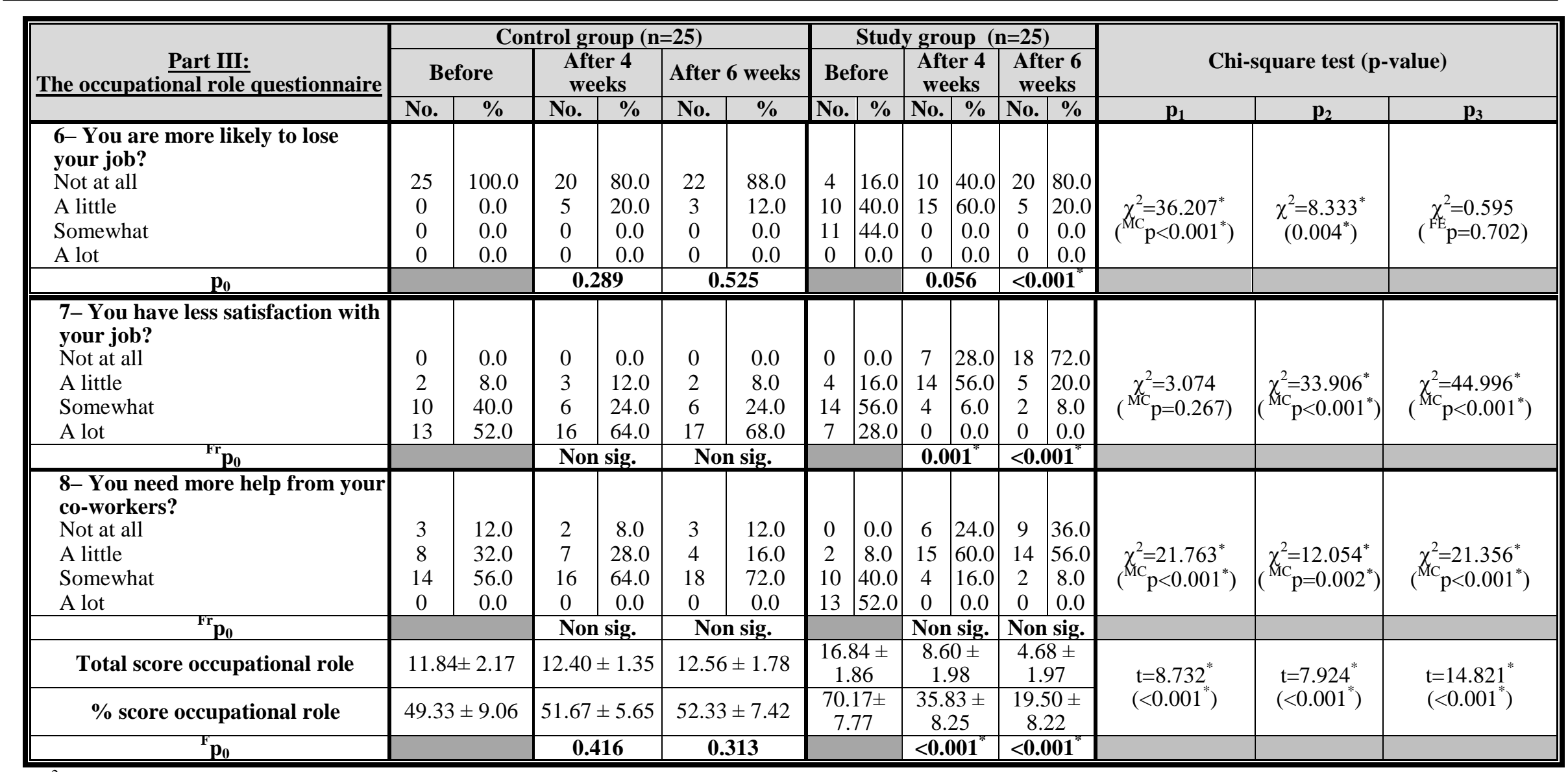

$\chi^{2}$ : Chi square test $\quad$ MC: Monte Carlo $\quad$ FE: Fisher Exact $\quad$ t: Student t-test

F: F test (ANOVA) with repeated measures, Sig. bet. periods was done using Post Hoc Test (Bonferroni)

Fr: Friedman test, Sig. bet. periods was done using Post Hoc Test (Dunn's) 
Abd-allah et al.,

Table (4): Comparison between the two studied groups according to the scoring system for occupational role

\begin{tabular}{|c|c|c|c|c|c|c|c|c|c|c|c|c|c|c|c|}
\hline \multirow{3}{*}{$\frac{\text { Scoring system for }}{\underline{\text { occupational tool }}}$} & \multicolumn{6}{|c|}{ Control group $(\mathrm{n}=25)$} & \multicolumn{6}{|c|}{ Study group $(\mathrm{n}=25)$} & \multirow{2}{*}{\multicolumn{3}{|c|}{ Chi-square test (p-value) }} \\
\hline & \multicolumn{2}{|c|}{ Before } & \multicolumn{2}{|c|}{$\begin{array}{c}\text { After } 4 \\
\text { weeks }\end{array}$} & \multicolumn{2}{|c|}{$\begin{array}{c}\text { After } 6 \\
\text { weeks }\end{array}$} & \multicolumn{2}{|c|}{ Before } & \multicolumn{2}{|c|}{$\begin{array}{c}\text { After } 4 \\
\text { weeks }\end{array}$} & \multicolumn{2}{|c|}{$\begin{array}{c}\text { After } 6 \\
\text { weeks }\end{array}$} & & & \\
\hline & No. & $\%$ & No. & $\%$ & No. & $\%$ & No. & $\%$ & No. & $\%$ & No. & $\%$ & $\mathbf{p}_{1}$ & $\mathbf{p}_{2}$ & $\mathbf{p}_{3}$ \\
\hline non interference & 0 & 0.0 & 0 & 0.0 & 0 & 0.0 & 0 & 0.0 & 10 & 40.0 & 24 & 96.0 & & & \\
\hline Partial interference & 24 & 96.0 & 25 & 100.0 & 23 & 92.0 & 5 & 20.0 & 15 & 60.0 & 1 & 4.0 & $\begin{array}{l}\chi^{2}=29.639 \\
\left(<0001^{*}\right)\end{array}$ & $\chi=12.50$ & $\begin{array}{c}\chi=54.545 \\
\mathrm{MC}_{\mathrm{n}} \leq 0.001^{*}\end{array}$ \\
\hline Complete interference & 1 & 4.0 & 0 & 0.0 & 2 & 8.0 & 20 & 80.0 & 0 & 0.0 & 0 & 0.0 & & $(<0.001)$ & $\left({ }^{2}<0.001\right)$ \\
\hline${ }^{\mathrm{Fr}} \mathbf{p}_{0}$ & & & \multicolumn{2}{|c|}{ Non sig. } & \multicolumn{2}{|c|}{ Non sig. } & & & \multicolumn{2}{|c|}{$<0.001^{*}$} & \multicolumn{2}{|c|}{$<0.001^{*}$} & & & \\
\hline
\end{tabular}

\section{$\chi^{2}$ : Chi square test MC: Monte Carlo}

Fr: Friedman test, Sig. bet. periods was done using Post Hoc Test (Dunn's)

$\mathrm{p}_{1}$ : $\mathrm{p}$-value comparing between control group and study group before

$\mathrm{p}_{2}$ : $\mathrm{p}$-value comparing between control group and study group after 4 weeks

$\mathrm{p}_{3}$ : $\mathrm{p}$-value comparing between control group and study group after 6 weeks

*: Statistically significant at $\mathrm{p} \leq 0.05$ 
Table (1): reveals that the majority of the control and study groups (80\%-92\%) were female and less than two third $(60 \%)$ of the control group and the majority of the study group $(76 \%)$ were between (21-30 years old). Also, the majority in both group $(68 \%, 80 \%)$ had a diploma degree. Concerning their marital status more than half of control group $(56 \%)$ and the majority of study group $(76 \%)$ were married and (56\%) of study group had (3-4) children whereas $(36 \%)$ of the control group had no child and (32\%) of them had (1-2) and (3-4) children. As regards to their body mass index (40\%) of the control group were normal and overweight while among the study group (72\%) had a normal weight. Also, the majority among both control and study groups were working in the morning shift, had no training about safety measure and had 6-10 years of experience ( $64 \%-80 \%)$, ( $92 \%-84 \%)$ and $(64 \%-72 \%)$ respectively. This table also showed that there was no statistically significant difference between both groups in relation to the socio-demographic characteristics.

Table (2): Among the study $40 \%$ had mild and moderate pain while $(64 \%)$ of control group had moderate pain. Also, (20\% - 8\%) of study and control group respectively had severe pain before acupressure. After 6 weeks of acupressure massage the majority $(76.0 \%)$ of the study group had no pain, while about two third $(64 \%)$ of the control group had moderate pain.

About two third $(64.0 \%)$ of the study group had moderate relief with pain killers before intervention. While after 6 weeks the majority (76.0\%) had whole pain relief. For the control group, about two third $(64.0 \%)$ had mild relief before and after 4 and 6 weeks.

As regard to back stiffness, about (68.0\%) of the study group had moderate and mild stiffness before intervention. While after 6 weeks the majority $(72.0 \%)$ of them had no stiffness. Compared with the control group, about two third $(64.0 \%)$ had mild stiffness before follow-up. While after 6 weeks the majority $(72.0 \%)$ of them still had mild stiffness.

The majority $(76.0 \%)$ of the study and control groups were unable to walk partially because of low back pain. While, after 4 and 6 weeks of acupressure the majority $(80.0 \%-88.0 \%)$ of the study group had complete freedom to walk as compared with $(20.0 \%-24.0 \%)$ of the control group respectively.

Nearly two third $(68.0 \%)$ of the study group and all $(100 \%)$ of the control were Sometime incapable of twisting while, after 4-6 weeks the majority of the study group had Complete freedom to twist as compared with only $(16.0 \%-12.0 \%)$ of the control group respectively.

Furthermore, the majority $(80.0 \%-84.0 \%)$ of the study and control group had more pain preventing them from sitting on such a chair before the program. After 4- 6 weeks the majority $(76.0 \%$ -
$84.0 \%$ ) of the study group had complete freedom to sit on a hard chair. While, all $(100.0 \%)$ of the control group had more pain when sitting on a chair after 4- 6 weeks.

About two thirds $(60.0 \%)$ of the study group had moderate pain when lying in bed before the program. While, after 4 weeks $(72.0 \%)$ had mild pain when lying in bed and after 6 weeks the majority had no back pain when lying in bed. For the control group, $64.0 \%-72.0 \%$ had mild pain when lying in bed before and after 4- 6 weeks of the program.

Concerning limitation in lifestyle functioning because of back pain, the majority $(72.0 \%)$ of the study group had moderate limitation when performing any task. While after 4 weeks the majority $(76.0 \%)$ of the study group had mild limitation when performing any task and after 6 weeks $84.0 \%$ was completely free from pain that allow them to perform any task. As regard the control group (48.0\%) before and after 4 weeks and $52.0 \%$ after 6 weeks were completely free from pain when performing any task. Moreover, $80.0 \%$ of the study group had sometime interference due to back pain. While after 4-6 weeks (64.0\% $88.0 \%)$ respectively had no interference at all. In the control group, $(60.0 \%)$ had some interference because of back pain.

As regards the need to modify their job, $64.0 \%$ of the study group needed a lot of adjustment on the job before the program and $(84.0 \%$ - 88.0\%) needed no adjustment of work after $4-6$ weeks of the program. Concerning the control group $100.0 \%$ - $96.0 \%$ - 84.0\%) needed no adjustment of work before and after $4-6$ weeks respectively.

There was a highly statistically significant difference before and after four and six weeks from application of acupressure program between study and control group $(\mathrm{P}=0.001)$.

Table (3): his table shows that the majority $(76.0 \%)$ of the study group usually cut down the extra work or over time due to LBP before the program. While After 6 weeks of acupressure massage the study group not need to cut down the extra work . compared with control group the majority of them usually cut down the extra work or over time. Also, (64.0\%) of the study group worked more slowly before the program a change nurses work ware observed to become less slowly after 6 weeks of the program. For control group were observed work more slowly throughout the follow up period.

More and more, for the study group (76.0\%) usually take more frequent or longer breaks. While after 6 weeks of acupressure massage about (36.0\%) of them not take more frequent or longer breaks. Concerning the control group usually take more frequent or longer breaks before and after 46 week throughout the follow up period. Also, $(80.0 \%)$ of the study group were somewhat less 
able to concentrate on their work before the acupressure massage. While $(68.0 \%)$ of the study group become more able to concentrate on their work after 6 weeks of acupressure massage. On the other hand, the majority of the control group was less able to concentrate on work throughout the follow up period.

Moreover, $(64.0 \%)$ of the study group had a lot fewer opportunities to upgrade their skills before the acupressure program due to low back pain, while more than half $(56.0 \%)$ of the study group had more opportunities to upgrade their skills after the low back pain disappeared by 6 weeks following acupressure massage. On the other hand, the majority of the control group had fewer opportunities to upgrade their skills throughout the follow up period. Furthermore, for the study group $(44.0 \%)$ were more likely to lose their job before the program, while the majority $(80.0 \%)$ of the study group were less likely to lose their job after 6 weeks of acupressure massage.

More than half $(56.0 \%)$ of the study group had less satisfaction with their job before acupressure massage while, $(72.0 \%)$ of them had higher satisfaction with their job after 6 weeks from acupressure. Also more than half $(52.0 \%)$ of the control group had less satisfaction with their job throughout the follow up period. More and more, $(52.0 \%)$ of the study group need more help from co-workers due to low back pain before the program. But after acupressure program and after disappearance of pain more than half of the study group needs little help from co-workers. While, the majority of control group still need more help from co-workers throughout the follow up period.

There was a highly statistically significant difference before and after application of acupressure program between study and control group $(\mathrm{P}=0.001)$.

Table (4): This table shows that $(80.0 \%)$ of the study group had back pain that completely interfere with their occupation before the program. While after 4 weeks $(60.0 \%)$ had partial interference and after 6 weeks $(96.0 \%)$ had no interference in occupation after acupressure massage. As regard to control group, (96.0\%-100.0\%-92.0\%) before and after $4-6$ weeks of the program respectively had partial interference in their occupation. And there was a highly statistically significant difference before and after four and six weeks from application of acupressure program between the study and control groups $(\mathrm{P}=0.001)$.

\section{Discussion}

Complementary medicine is in a remarkable place today, with one out of every three people using it at some point in their lives. Acupressure is a type of complementary and alternative medicine. It is noninvasive, low-cost, needs no guidance for administration, is affordable compared to other medical methods, and is finally easy to comprehend (Sadri et al., 2006). The acupressure technique is based on points that are pressed with a finger; this pressure induces endorphin release, leading to relaxation of the muscle and minimizing pain.

The result of the present study revealed that the mean age groups $( \pm \mathrm{SD})$ of the control group nurses were $33.68 \pm 7.26$ years, while in the study group nurses were $33.96 \pm 6.59$ years compared with less experienced and younger nurses. This finding is consistent with (Bejia et al., 2005 and Hinmikaiye \&Bamishaiye 2012), who reported that being a female nurse was significantly associated with occurrence of low back pain. Also, (Mwilila et al., 2008) reported that female nurses made up 83.6 percent of the total study population and that they had the highest prevalence of low back pain as compared to their male counterparts. More and more, (Movahedi, et al., 2017) investigated the influence of acupressure on pain severity among female nurses with chronic low back pain and found that the mean age (SD) of the participants was 34.007 .58 years, with a mean experience of 12.167 .22 years.

Furthermore, (Abou El-Soud et al.,2014, Bastani et al 2012, and Momenabadi, 2010) mentioned that acupressure is an effective complementary treatment to alleviate low back pain in nursing workers, increase pain ratings and shift pain from moderate to low and have a beneficial effect on pain relief. Also, (Sorour et al., 2014) and (Amany et al. 2014) revealed that acupressure party experienced a substantial reduction in pain after the intervention. Again (Mahmodzadeh, 2012) noted that acupressure at particular points minimized low back pain. (Karimipour et al. 2012) declared no beneficial impact of acupressure on pain severity in Rheumatoid Arthritis patients. Low back pain has a direct impact on job quality of life, the overburdens on the healthcare system, and owing to the high rate of absenteeism. In light of this statement, the findings of this study revealed that the acupressure technique has a reasonable impact on the study group compared to the control group in terms of decreased back stiffness, decrease rate of absenteeism among nurses, decreased back pain that interferes with freedom of movement, ability to stand, and decreased back pain. So, the improvement occurs in the study group than the control group with a statistically significant difference between pre and post- intervention. According to (Mahmodzadeh, 2012\&Zhang et al., 2012), acupressure massage on specific points was found to be effective in reducing pain and improving the overall occupaThe current study's findings revealed that acupressure massage improves the study group's occupational function compared to the control group.Also, there was a statistically significant difference between pre and post- intervention. These results go in the same line 
with, Moussa et al., 2015; Hinmikaiye \& Bamishaiye, 2012, Wong et al., 2010 and Purepong et al. (2015) who found that acupressure is a cheap and non-invasive method that causes endorphin release, which leads to muscle relaxation and pain relief.

The post-test mean score of occupational role in the study group was $19.50 \pm 8.22$, while in the control group it was $52.33 \pm 7.42$, with a highly statistically significant difference between the two groups at $\mathrm{p}$ $\left(0.001^{*}\right)$. The findings of this study agree with those of (Hsieh, et al 2004 ), who discovered a substantial reduction in low back pain and disability among female school teachers who received acupressure, with the post-test mean score of disability in the study group being $16.33 \pm 6.47$ and the control group $52.10 \pm 3.242$, which was statistically significant at $\mathrm{P} 0.001$.

Finally, as a complementary effective treatment, acupressure has been shown to reduce occupational low back pain in nurses, and change pain from moderate to low. The findings contributed to a better understanding of acupressure massage, the recognition of low back pain triggers, and the assessment of massage as a possible future treatment for nurses.

\section{Conclusion:}

Based on the results of this study, it can be concluded that acupressure at a specific point is successful in reducing low back pain and improving the function of nurses.

\section{Recommendation}

- Further experiments with a larger sample size and more follow-up sessions are required to obtain more generalized findings.

- Educational nursing program about effect and technique of acupressure.

\section{References:}

- Abou El-Soud, A. M., El-Najjar, A. R., ElFattah, N. A., \& Hassan, A. A. (2014). Prevalence of low back pain in working nurses in Zagazig University Hospitals: an epidemiological study. Egyptian Rheumatology and Rehabilitation, 41(3), 109.

- Amany, S, Amany, A \& Eman AEA (2014), 'Effectiveness of acupressure versus isometric exercise on pain, stiffness, and physical function in knee osteoarthritis female patients', Journal of Advanced Research, vol. 5, no. 2, pp. 193-200. doi: 10.1016/j. -jare.2013.02.003

- Bastani FM, Sobhani M, Bozorgnejad S, ShamsikhaniHaghani H (2012). Effect of acupressure on severity of pain in women with multiple sclerosis (MS) Complement Med J. 2:75-84.

- Bejia, I., Younes, M., Jamila, H. B., Khalfallah, T., Ben Salem, K., Touzi, M.,
Akrout, M., Bergaoui, N (2005). Prevalence and factors associated to low back pain among hospital staff. Joint Bone Spine, 72, 254-259.

- Bono JP, Hudsmith LE, Bono de A (2001). Back pain in pre-registration house officers. Occupational Medicine; 51(1):62-65.

- Chen YW, Wang HH (2014). The effectiveness of acupressure on relieving pain, a systematic review. Pain ManagNurs. 15(2):539-50. doi: 10.1016/j.pmn.2012.12.005. [PubMed: 23415783].

- Chen, H. M., Wang, H. H., Chiu, M. H., \& Hu, H. M. (2015). Effects of acupressure on menstrual distress and low back pain in dysmenorrheic young adult women: an experimental study. Pain Management Nursing, 16(3), 188-197.

- Dabiri G., \& Kaya N. (2019). Use of Acupressure for Women's Health. Recent Studies in Health Sciences, 54, 324

- Hinmikaiye, C. D., \&Bamishaiye, E. I. (2012). The incidence of low back pain among theatre nurses: a case study of university of Ilorin and ObafemiAwolowo University Teaching Hospital.International Journal of Nursing Science, 2(3), 23-28.

- Hsieh, L. L. C., Kuo, C. H., Yen, M. F., \& Chen, T. H. H. (2004). A randomized controlled clinical trial for low back pain treated by acupressure and physical therapy. Preventive medicine, 39(1), 168-176.

- HuJohnson, P (2007). Global use of complementary and alternative medicine (CAM) and treatments. Global health care: Issues and policies, 199.

- Kamper, S. J., Apeldoorn, A. T., Chiarotto, A., Smeets, R. J., Ostelo, R. W., Guzman, J., \& Van Tulder, M. W. (2015). Multidisciplinary biopsychosocial rehabilitation for chronic low back pain: Cochrane systematic review and meta-analysis. Bmj, 350.

- Karimipour, F., Fayazi, S., Mowla, K., \& Latifi, S. M. (2012). Effect of acupressure on severity of pain in arthritis rheumatoid patient. Jundishapur Scientific Medical Journal, 11(3), 269-275.

- Kopec, J. A., \& Esdaile, J. M. (1998). Occupational role performance in persons with back pain. Disability and rehabilitation, 20(10), 373-379.

- Million, R. Hall ,W. Nilsen, K.H. Baker ,R.D. Jayson ,M.I. (1982). Assessment of the progress of the back-pain patient 1981 Volvo Award in Clinical Science. Spine (Phila Pa 1982 (7),204-12.

- Mohamannazhad, A., Abotalabi, G., \& GHaforifard, M. (2012). Effect of acupressure on low back pain in patients with spondylarthritis. Journal of Urmia Nursing \& Midwifery Faculty, 10(2). 
- Moemenabadi, H (2010). Examining the effect of acupressure on the intensity of pain and physiologic indicators resulting from removal of chest drainage tube at ICU of Shariati Hospital (Persian)], MA thesis, Iran University of Medical Sciences.

- Moussa, M. M. M., El-Ezaby, H. H., \& ElMowafy, R. I. (2015). Low back pain and coping strategies' among nurses in Port Said City, Egypt. Journal of Nursing Education and Practice, 5(7), 55-62.

- Movahedi, M., Ghafari, S., Nazari, F., \&Valiani, M. (2017). The effects of acupressure on pain severity in female nurses with chronic low back pain. Iranian Journal of Nursing and Midwifery Research, 22(5), 339342.

- Mwilila, M.C (2008). Work-related low back pain among clinical nurses in Tanzania. Physiotherapy department, University of the Western Cape.

- Purepong, N., Channak, S., Boonyong, S., Thaveeratitham, P., \& Janwantanakul, P. (2015). The effect of an acupressure backrest on pain and disability in office workers with chronic low back pain: A randomized, controlled study and patients' preferences. Complementary Therapies in Medicine, 23(3), 347-355.

- Sadri, B, Nematullahi, M, \&Shahrami, R (2006). '[The effect of acupressure on reducing nausea and vomiting after adenotonsillectomy (Persian)]', Journal of Iran University of Medical Sciences. 13(53): 121-26.

- Salzberg LD, Manusov EG(2013). Management options for patients with chronic back pain without an etiology. Health Serv Insights;6:33-8.

- Sharafkhani, N., Khorsandi, M., Shamshi, M., \&Ranjbaran, M. (2014). Low back pain preventive behaviors among nurses based on the health belief model constructs. SAGE Open, 2014, 1-7.

- Sorour A, Amany S. Ayoub b,and Eman M. Abd El Aziz. (2014) Effectiveness of acupressure versus isometric exercise on pain, stiffness, and physical function in knee osteoarthritis female patients. Journal of Advanced Research 5, 193-200

- Tinubu, B. M., Mbada, C. E., Oyeyemi, A. L., \& Fabunmi, A. A. (2010). Work-related musculoskeletal disorders among nurses in Ibadan, South-west Nigeria: a cross-sectional survey. BMC Musculoskeletal disorders, 11(1), 12.

- Tosunoz, I. K., \&Oztunc, G. (2017). Lower back pain in nurses. International Journal of Caring Sciences, 10(3), 1728-1732.

- Wong, T. S., Teo, N., \& Kyaw, M. O. (2010). Prevalence and risk factors associated with low back pain among health care providers in a district hospital. Malaysian Orthop Journal, 4(2), 23-28

- World Health

Organization

[WHO]/Osteoarthritis/census;

2007.<http://www.Who.ORG>.

- Yeh CH, Chien LC, Chiang YC, Huang LC(2012). Auricular point acupressure for chronic low back pain, a feasibility study for 1week treatment. Evid Based Complement Alternat Med

- Zhang Y, Shen CL, Peck K, Brism' ee JM, Doctolero S, Lo DF, et al (2012). Training self-administered acupressure exercise among postmenopausal women with osteoarthritic knee pain: a feasibility study and lessons learned. Evidence-Based Complement Altern Med.2012:570431, http://dx.doi.org/ $10.1155 / 2012 / 570431$. 